\title{
La plataforma Edmodo como recurso didáctico en la enseñanza de la escritura académica del idioma inglés
}

\section{The Edmodo platform as a teaching resource in the teaching of academic writing of the english language}

Piedad Gisela Mejía Gavilánez

Karla Paulina Hidalgo Montesinos

Andrea de los Ángeles Rosero Morales

William Eduardo Yugsan Gómez

Universidad Central del Ecuador, Ecuador

Autora para correspondencia: pgmejia@uce.edu.ec/pmejiagavilanez83@hotmail.com Fecha de recepción: 04 de Diciembre 2017 - Fecha de aceptación: 15 de Junio de 2018

Resumen: La enseñanza- aprendizaje del idioma inglés ha sido altamente destacada en los últimos años, y con ello se ha generado el Nuevo Rediseño de la Carrera de Pedagogía de los Idiomas Nacionales y extranjero-inglés de la Universidad Central del Ecuador aprobado por el CES en el año 2016 con el objetivo de cumplir importantes expectativas en este campo. Es por esta razón que los educadores no pueden quedarse fuera del desarrollo científico y tecnológico. El presente artículo sustenta la aplicación de la plataforma Edmodo como recurso didáctico en la enseñanza de la escritura académica en los y las estudiantes de tercer semestre de la Carrera Inglés. Los datos presentados indican la posibilidad de la propuesta para responder a los problemas encontrados en la Carrera de Inglés, la misma que se respalda en una investigación cuasi-experimental, con métodos y técnicas de investigación adecuados durante el desarrollo del proyecto. Finalmente, los estudiantes encuentran ventajas en la integración de los medios didácticos basados en la plataforma Edmodo para desarrollar la precisión, la brevedad y la claridad.

Palabras Claves: plataforma Edmodo; escritura académica; desarrollo; cuasi-experimental

\begin{abstract}
The teaching-learning of the English language has been highly highlighted in recent years, and this has generated the New Redesign of Pedagogy of the National Languages and foreign-English Major at Central University of Ecuador approved by the CES in the year 2016 with the aim of fulfilling important expectations in this field. It is for this reason that educators cannot be left out of scientific and technological development. This article supports the application of the Edmodo platform as a didactic resource in the teaching of academic writing in the third semester students at English Major. The presented data indicate the possibility of the proposal to respond to the problems found in the English Major, which is supported by a quasi-experimental research, with appropriate research methods and techniques during the development of the project. Finally, students find advantages in the integration of didactic media based on the Edmodo platform to develop accuracy, brevity and clarity.
\end{abstract}

Key Words: Edmodo platform; academic writing; development; quasi-experimental 


\section{Introducción}

La educación se vuelve cada vez más demandante, exigente y competitivo, para ello se requiere del apoyo de recursos que ayuden en el proceso de enseñanza- aprendizaje de los estudiantes de la Carrera de Inglés. Los materiales didácticos, su uso tiende a guiar y motivar al universitario en la construcción del conocimiento, es decir, que sirvan de apoyo en el proceso de conocimiento de los educandos mediante publicaciones de sistemas pedagógicos innovadores utilizando herramientas tecnológicas.

Uno de los problemas que se han enfrentado los estudiantes de la Carrera de Inglés y las instituciones educativas no ha sido la integración de las TICs a los procesos de enseñanzaaprendizaje, porque una vez que se posee la tecnología, el tema que surge es cómo los futuros profesores pueden hacer uso de ella, y de qué manera la van a integrar a los métodos de enseñanza.

Según Mumtag (2005), los principales factores que influyen en el uso de las TICs por parte de los docentes son: el acceso a este tipo de recursos, calidad de software y equipos, facilidad o simplicidad de uso, incentivo para cambiar las prácticas pedagógicas usando tecnología, el apoyo y solidaridad de las instituciones para usar las TICs en el currículo, las políticas nacionales y locales sobre TICs, compromiso con la superación profesional, y la capacitación formal recibida en el uso de las TICs. (Gallardo \& Buleje, 2010).

Según Sánchez (2014), un material didáctico es el conjunto de medios materiales que intervienen y facilitan el proceso de enseñanza-aprendizaje, pueden ser tanto físicos como virtuales, que despiertan el interés y capten la atención de los educandos. Al mismo tiempo los materiales didácticos presentan información adecuada con experiencias simuladas cercanas a la realidad, que vivifican la enseñanza influyendo favorablemente en la motivación, retención y comprensión por parte del discípulo, facilitando la labor docente por ser sencillos, consistentes y adecuados a los contenidos.

\section{Desarrollo}

La evolución de las tecnologías de la información y comunicación plantea nuevos desafíos en la educación, razón por la cual el empleo de la Plataforma Edmodo permite el avance de la escritura académica de los y las estudiantes de tercer semestre de la Carrera Inglés de la Facultad de Filosofía de la Universidad Central del Ecuador, lo que significa desarrollar en los educandos los conocimientos, hábitos y habilidades básicas que le permitan comunicarse en la lengua inglesa. Entonces se hace necesario que en el trabajo en el aula además de presentar modelos, realizar ejercicios, se utilice la Plataforma Edmodo en el aula, está ayudaría en gran parte a resolver los problemas en la enseñanza del inglés, donde se puede encontrar diferentes recursos para enseñar la escritura académica, como por ejemplo enviar mensajes, tareas, cuestionarios, anotar actividades en el calendario o libro de calificaciones y almacenar documentos o archivos en la biblioteca. El estudiante aprenda de una manera entretenida, creativa y más dinámica con la utilización de estos recursos.

En la Universidad de Valladolid (España) un estudio sobre "Plataforma Edmodo versus la plataforma Moodle "realizado como fin de maestría por Paz (2013), donde manifiesta lo siguiente: "Edmodo y Moodle son herramientas que tratan de ser para los alumnos una fuente de motivación que 
favorezca la construcción de su saber y les permita aprender en entornos digitales" Pag. 66. Se puede decir que ambas plataformas son herramientas de uso donde el estudiante se siente motivado para aprender. Estas plataformas tienen consideraciones pedagógicas generales que los usuarios pueden explorar y desarrollar en sus propios usos educativos.

Además, la funcionalidad de estas plataformas tendrá sentido cuando se integren en una actividad, de este modo cada docente o persona que las utilice podrá construir su propia experiencia. Este nuevo rol no disminuye la importancia del docente, pero requiere de nuevos conocimientos y habilidades. Los estudiantes serán más responsables de su propio aprendizaje en la medida en que busquen, encuentren, sinteticen y compartan su conocimiento con otros compañeros. La Plataforma Edmodo constituye una herramienta poderosa para apoyar este cambio y para facilitar el surgimiento de nuevos roles en docentes y estudiantes.

Por ejemplo, se puede subir archivos y videos académicos de las páginas web www.owl.english.purdue.edu/engagement/2/1/29/; www.englishaula.com; www.flo joe.com; www.TED talks; www.youtube.com/watch?v=-NYFIFgrQog_a la plataforma Edmodo con el fin de que el estudiante pueda realizar actividades para el desarrollo de la escritura académica del idioma inglés de manera individual o grupal dependiendo del tipo de tarea.

El desarrollo de habilidades para escribir textos académicos (...) exige un entrenamiento de alta complejidad (...). Escribir, en este nivel, es una actividad social que implica interpretar la información de manera crítica, integrar información obtenida en fuentes diversas, crear información, proponer nuevos enfoques sobre aspectos teóricos ya desarrollados y justificarlos con argumentos, validar teorías con investigaciones empíricas o refutar otras con nuevos datos o nuevas interpretaciones de datos existentes... (Cubo de Severino, L., 2007, p. 15).

Green (2000), New (1999) e Hirvela (2005), por su parte, también realizaron estudios sobre la escritura académica en entornos computacionales. Green diseñó un curso de escritura en inglés como lengua extranjera mediado por computador para estudiantes universitarios japoneses, basándose en el enfoque de la escritura como proceso. El investigador planteó un énfasis en los patrones de organización presentes en los textos dados como modelos y en los propósitos de redacción de cada sujeto. De otro lado, observando las estrategias de revisión empleadas por estudiantes de francés en dos tareas de redacción utilizando el software Système D, New encontró que los educandos revisaron sus textos predominantemente a nivel de la superficie textual sin alterar la estructura ni el sentido global; también constató un incremento de la revisión gráfica en los textos escritos en el computador, pero un uso insuficiente de las herramientas de referencia ofrecidas por el software.

Finalmente, Hirvela encontró que las prácticas de lectura y de escritura electrónicas en L2 varían considerablemente entre un curso y otro y que, en muchas materias, la escritura juega un papel importante para la valoración del conocimiento académico y del desempeño de los estudiantes. Hirvela resalta la importancia de 356 Universidad del Valle Martha Berdugo T. - Oscar Eduardo Herrera Verónica Valdiri V. trabajar sistemáticamente algunos géneros empleados como los ensayos problemasolución, las reseñas y los textos de opinión que requieren citación y habilidades de pensamiento crítico durante el proceso de composición.

Dentro de este marco, es necesario plantear las hipótesis: 
- Hi: La aplicación de la plataforma Edmodo influye en el desarrollo de la escritura en los y las estudiantes de tercer semestre de la Carrera Inglés de la Universidad Central en el periodo 2015.

- Ho: La aplicación de la plataforma Edmodo no influye en el desarrollo de la escritura en los y las estudiantes de tercer semestre de la Carrera Inglés de la Universidad Central en el periodo 2015.

La justificación del presente proyecto nace a raíz de la siguiente interrogante ¿Por qué los y las estudiantes que terminan los estudios secundarios tienen una limitada expresión escrita en el idioma inglés?, todo esto se debe a factores condicionantes que según Nelson Martínez manifiesta lo siguiente" Hay al menos cinco factores que son determinantes en el proceso enseñanza- aprendizaje del idioma inglés: el docente, el estudiante, la metodología, el currículo y el contexto educativo. Estos factores en condiciones favorables pueden llevar a los estudiantes a desarrollar mejores niveles de aprendizaje, pero en circunstancias desfavorables pueden interferir y afectar los resultados grandemente." (Pag. 7.)

Con relación a lo expuesto se puede decir que los factores condicionantes para el proceso de enseñanza-aprendizaje del idioma inglés son importante porque, el estudiante encuentra un contexto social, cultural y lingüístico en el que inglés está apenas presente para interactuar y sobrevivir en ese contexto.

A partir de esto y conociendo la importancia del inglés en la actualidad, es importante enfocarse en este problema donde hoy en día el uso de la plataforma Edmodo es de gran importancia, según los estudios realizados en la Universidad de Valladolid en el uso de la plataforma Edmodo versus la plataforma Moodle realizado por María Paz donde manifiesta lo siguiente " La plataforma Edmodo propone actividades y plantea secuencias didácticas que pueden conllevar aprendizajes tales como el aprender nuevos conceptos, buscar información, publicar creaciones propias, la mejora a través de la revisión de los textos o el feedback con los otros participantes.” (Pag. 15.)

Con relación a lo mencionado se puede decir que el docente deja de ser un mero transmisor de conocimientos desempeñando el rol de mediador o facilitador de los procesos de aprendizaje, cuya principal meta es la transformación en conocimiento de la información. La plataforma Edmodo es una herramientas informática que ayuda a desarrollar la expresión escrita del idioma inglés, y más aún con el gran auge de la tecnología, en donde casi en su totalidad la información se encuentra en inglés; por ello la trascendencia que los/las estudiantes interpreten y apliquen el idioma inglés en situaciones comunes de comunicación escrita en las diferentes facetas y roles de la vida del ser humano.

De igual manera, según el estudio realizado por la organización de educación más grande del mundo EF-EPI (2017) manifiesta que el idioma inglés es esencial para la comunicación internacional, es el idioma de la ciencia, de los negocios y de la diplomacia, por tal efecto es una manera profunda de interconectividad en el mundo.

Los resultados del Ranking Mundial más grande del dominio del inglés estima que el $52 \%$ de los principales 10 millones de sitios de Internet están en inglés. El dominio del inglés permite que los usuarios cotidianos de Internet tengan acceso a estos recursos y compartan sus ideas y experiencias con una audiencia más amplia. Encontramos que los niveles más altos de 
uso de Internet se correlacionan fuertemente con un mayor dominio del inglés (Gráfico 1.), así como las medidas de acceso a Internet tales como suscripciones a banda ancha y la cantidad de servidores seguros (Gráficos 2 y 3). Un mayor acceso a Internet proporciona una mayor exposición al idioma inglés, lo que incrementa el grado de dominio de la lengua inglesa. A su vez, habilidades en inglés más sólidas facilitan el acceso a aún más recursos en línea en esta lengua (EF EPI, 2017).

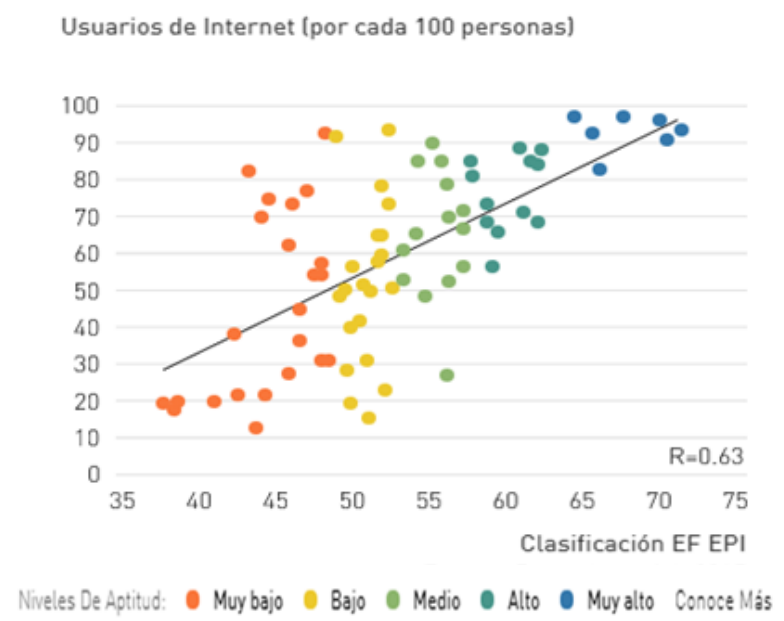

Gráfico 1. El inglés y los usuarios de internet. Fuente: Banco Mundial, 2015

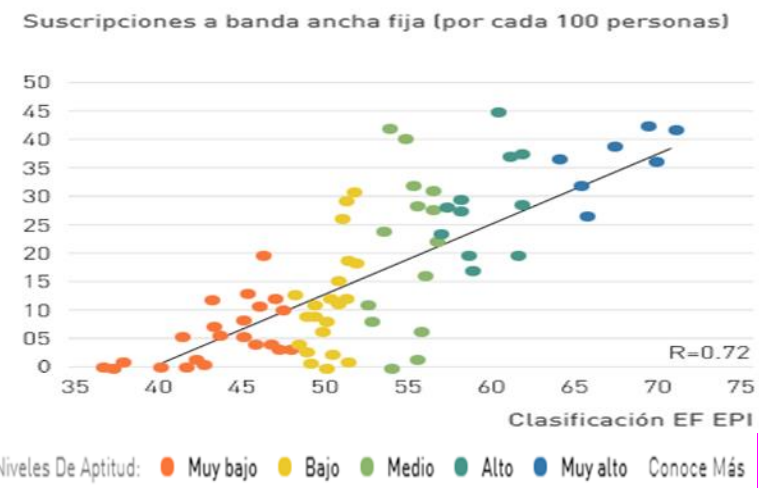

Gráfico 2. El inglés y el acceso a banda ancha Fuente: Banco Mundial, 2015

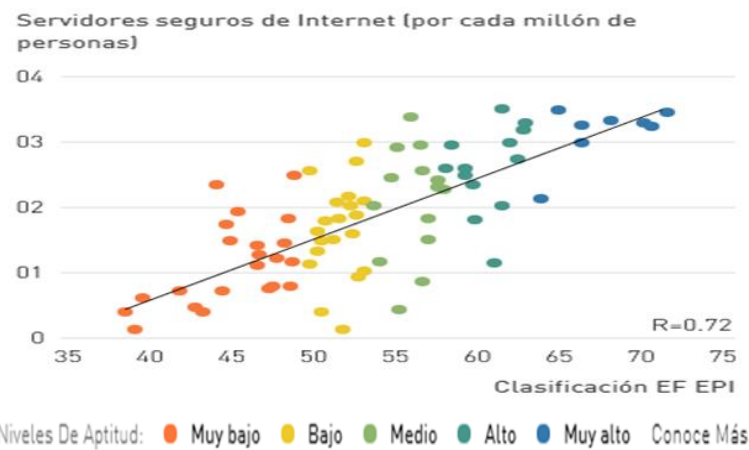


Gráfico 3. El inglés y la seguridad en internet

Fuente: Banco Mundial, 2015

Por ello se hace necesario, que los docentes, estudiantes y padres de familia pueden explotar al máximo las herramientas de la plataforma Edmodo de manera segura y fácil, no obstante, cuenta con características intuitivas y almacenamiento ilimitado, podrá crear rápidamente grupos, asignar tareas, programar su envío, gestionar el progreso y mucho más, todo en una sola plataforma.

La Plataforma Edmodo es una herramienta en el proceso de aprendizaje, por lo que es esencial que tanto los futuros docentes como los docentes en actividad sepan utilizarla y servir como modelo para la capacitación en lo que respecta a nuevos métodos pedagógicos y nuevas herramientas de aprendizaje. Por otro lado, aprender un idioma significa aprender a usarla, y si el estudiante ya la conoce significa comunicarse mejor y en situaciones complejas. El educando también tiene que conocer el vocabulario académico, puntuación y modelos de escritura para que se convierta en un experto al momento de comunicarse de forma escrita.

Las interacciones que se realizó con el grupo control fue de revisar la plataforma todos los días y cumplir con las tareas de: escribir diálogos o párrafos cortos de 60 palabras y dar opiniones o comentarios de tres compañeros durante todos los tres meses y en el aula se realizaba la retroalimentación. En los dos siguientes meses se realizó los procesos de escritura donde involucro el estudio de un párrafo, los tipos, las estructura, la coherencia, para finalmente redactar cartas, correos y ensayos. Pasado ese tiempo, el estudiante estuvo en la capacidad de escribir párrafos largos de 120 a 150 palabras con la utilización de los conectores, la puntuación, el uso de las abreviaturas previamente revisados y practicados en la clase y con el apoyo de sus compañeros al momento de escribir sugerencias o consejos para mejorar y reforzar sus conocimientos en la plataforma.

Pérez C. (2013) manifiesta lo siguiente: Todos los niveles de nuestra formación se desarrollan en medio de textos: en la escuela, en el colegio, en la Universidad. Ejercicios, exámenes, ensayos, informes, tesis exigen trabajos de escritura. Por lo tanto, el conocimiento y la aplicación de las posibilidades expresivas de la lengua son indispensables para la formación del futuro profesional universitario (pág. 6) Con relación a lo expuesto se puede decir que en todo ámbito educativo se requiere trabajos de escritura, por lo que cada persona expresa las ideas con el fin de comunicarse.

Finalmente, como objetivos de la investigación se proyectó analizar la aplicación de la plataforma Edmodo en el desarrollo de la escritura académica en los y las universitarios de tercer semestre de la Carrera Inglés de la Universidad Central y la promoción del proceso de comunicación y transmisión de información virtual en la muestra dentro de los contextos educativos formales.

\section{Métodos}

Esta investigación pertenece a un diseño cuasi-experimental en la que se ha tratado como variable independiente (Plataforma Edmodo) que es la causa, para observar el efecto con la variable dependiente (Escritura Académica), los grupos no se han asignado al azar, ya estaban conformados previamente, en este caso, como paralelos: "A" $\mathrm{y}$ “ $\mathrm{B}$ ”. Este diseño tiene un grupo 
experimental, un grupo de control y con mediciones "antes" (pre-test) y "después" (post-test) en ambos grupos.

Según Hernandez y otros (2006:159) es cuasi-experimental cuando “...los sujetos no son asignados al azar a los grupos, ni emparejados; sino que dichos grupos ya estaban formados antes del experimento, son intactos..." Con el propósito de aplicar una pre-prueba para diagnosticar el nivel de desarrollo de producción escrita de los alumnos del tercer semestre de la Carrera de Inglés, y posteriormente aplicar una post-prueba para determinar si las actividades realizadas a través de la plataforma Edmodo influyen en el proceso de aprendizaje, específicamente en la producción escrita en inglés de los estudiantes anteriormente mencionados. (Piña, 2009).

El enfoque realizado fue cuali-cualitativo, es decir, se combinó los dos enfoques en todo el proceso de investigación. El punto de vista Cuantitativo recogió, procesó y analizó datos y números sobre variables determinadas, ya que se ha realizado la recolección de reseñas a través de una encuesta aplicada a los y las estudiantes, además se aplicó el pre-test y el post-test a las y los universitarios de dos paralelos para posteriormente realizar el respectivo análisis estadístico e interpretación de los datos. La examinación de carácter Cualitativo describió la realidad tal como la experimentaron los estudiantes.

Para Hernández, R., Fernández, C., y Baptista, P., (2010) el enfoque cualitativo "Utiliza la recolección de datos sin medición numérica para descubrir o afinar preguntas de investigación en el proceso de interpretación". (p. 7). Y el enfoque cuantitativo "Usa la recolección de datos para en base a la medición numérica y el análisis estadísticos, para establecer patrones de comportamiento y probar teorías". (p.4).

Esta investigación fue Aplicada porque se sintió la necesidad de resolver la dificultad encontrada en el desarrollo de la escritura académica. Zorrilla (1993) la investigación aplicada "Depende de los descubrimientos y avances de la investigación básica y se enriquece con ellos, pero se caracteriza por su interés en la aplicación, utilización y consecuencias prácticas de los conocimientos. La investigación aplicada busca el conocer para hacer, actuar, construir y modificar". (p. s/n)

También es documental porque reúne, selecciona y analiza datos que se encuentra en documentos escritos ya sean impresos, electrónicos o audiovisuales hechos por el hombre, los cuales fueron de ayuda en la investigación. Como Alfonso. (1995) quien es citado por Morales., O. (s/a) señala que la investigación documental "es un procedimiento científico, un proceso sistemático de indagación, recolección, organización, análisis e interpretación de información o datos en torno a un determinado tema. Al igual que otros tipos de investigación, éste es conducente a la construcción de conocimientos" ( $\mathrm{s} / \mathrm{p})$.

Es considera de campo como menciona Arias, (1999) porque "Consiste en la recolección de datos directamente de la realidad donde ocurren los hechos, sin manipular o controlar alguna variable" (pág. 19). Es decir, la investigación de campo es beneficiosa porque se recoge los datos en lugar donde suceden los hechos sin dar tiempo a manipular los datos que se obtienen. 
Es de tipo descriptiva porque busca medir conceptos o variables; así como, evaluar distintos aspectos de un universo con la finalidad de identificar características o establecer propiedades importantes que permitan informar sobre el fenómeno estudiado. Es decir, abordó el relacionamiento de la teoría con la práctica porque indagó la explicación de cualidades, características de los grupos u objetos sometidas a estudio, análisis y ajuste lógico generador de las respectivas conclusiones y recomendaciones.

La investigación involucró a 60 universitarios de los terceros semestres de la Carrera Inglés, de los cuales 30 estudiantes pertenecen al grupo experimental y 30 similares conforman al grupo control. Se trabajó con dos cursos correspondientes a los paralelos "A" y "B". Los sujetos se caracterizaron por ser jóvenes entre 18 y 21 años de edad. En su mayoría provienen de hogares de clases económicas y culturales medias bajas.

\section{Resultados}

Se elaboró una matriz de datos, diferentes cálculos estadísticos necesarios y se generaron tablas y gráficos en el programa SPSS que facilitaron el análisis e interpretación de las reseñas recolectados sobre las pre y post- pruebas de la Plataforma Edmodo aplicados a los y las estudiantes pertenecientes a los paralelos "A" y "B" de la Carrera.

IBM SPSS Statistics (s/f) es el software estadístico líder, utilizado para resolver una gran variedad de problemas de negocio e investigación. Proporciona distintas técnicas, incluyendo el análisis ad-hoc, pruebas de hipótesis e informes, para facilitar la gestión de datos, seleccionar y realizar análisis y compartir los resultados. La solución está disponible como opción de suscripción o licencia perpetua.

Además, se utilizó la prueba t para realizar el análisis comparativo de los datos de las pre y post- pruebas aplicadas a las y los estudiantes del grupo experimental y grupo control. Este ensayo se emplea para evaluar la diferencia significativa entre las medias aritméticas de dos grupos o dos categorías. Esta tentativa también proporciona resultados aproximados para medias aritméticas.

Sánchez R. (2015) La prueba t-Student se fundamenta en dos premisas; la primera: en la distribución de normalidad, y la segunda: en que las muestras sean independientes. Permite comparar muestras, $\mathrm{N} \leq 30 \mathrm{y} / \mathrm{o}$ establece la diferencia entre las medias de las muestras. El análisis matemático y estadístico de la prueba con frecuencia se minimiza para $\mathrm{N}>30$, utilizando pruebas no paramétricas, cuando la prueba tiene suficiente poder estadístico.

En la prueba para una media poblacional con muestra pequeña y desviación estándar poblacional desconocida se utiliza el valor estadístico t.

Análisis comparativo de los resultados del pre y pos-test aplicada a los y las estudiantes mediante la prueba $\mathrm{T}$

Tabla 1. Prueba t para medias de los muestras grupo experimental

$$
\text { Prueba t para medias de dos muestras emparejadas del grupo experimental }
$$




\begin{tabular}{lll}
\hline & Variable 1 & Variable 2 \\
Media & 15 & 27,68 \\
Varianza & 72,5 & 63,97 \\
Observaciones & 29 & 29 \\
Coeficiente de correlación de Pearson & $-0,0171752$ & \\
Diferencia hipotética de las medias & 0 & \\
Grados de libertad & 28 & \\
Estadístico t & $-5,79624989$ & \\
$\mathrm{P}(\mathrm{T}<=\mathrm{t})$ una cola & $1,5854 \mathrm{E}-06$ & \\
Valor crítico de t (una cola) & 1,70113093 & \\
$\mathrm{P}(\mathrm{T}<=\mathrm{t})$ dos colas & $3,1708 \mathrm{E}-06$ & \\
Valor crítico de t (dos colas) & 2,04840714 & \\
\hline
\end{tabular}

Fuente: Prueba de la escritura académica

Elaborado por: Piedad Mejía

Como se puede observar en este cuadro, se han realizado varios cálculos mediante la aplicación de la prueba $\mathrm{T}$, uno de ellos es la Media donde se aprecia que existe una diferencia de 12,68 puntos en el resultado de la aplicación de la pre y post-prueba, este grupo de estudiantes mejoraron el desarrollo de la escritura académica con el uso de actividades diseñadas con la Plataforma Edmodo.

Al comparar la media aritmética obtenida en los dos tipos de pruebas y en cada uno de los grupos, se observa que el incremento de respuestas acertadas en el grupo experimental en relación al grupo control es de $\mathbf{3 , 1 2}$.

Tabla 2. Resultados de la media aritmética

\begin{tabular}{llll}
\hline \multicolumn{2}{l}{ Resultados de la media aritmética } & & \\
\hline \multirow{2}{*}{ Grupo Experimental } & Pre-prueba & Post-prueba & Diferencia \\
Grupo control & 27,99 & 39,07 & 11,08 \\
Total & 26,73 & 34,49 & 7,76 \\
\hline
\end{tabular}

Fuente: Prueba de la escritura académica

Elaborado por: Piedad Mejía

En esta tabla se puede observar que existe una media aritmética de 3,32 de diferencia entre grupo experimental y grupo control. Finalmente de acuerdo a los resultados presentados tanto en el pre-test y en el post-test de la t de student y de la media aritmética se acepta la hipótesis alternativa y se rechaza la hipótesis nula.

Ramos, C. (2012) manifiesta que la hipótesis nula es aquella que nos dice que no existen diferencias significativas entre los grupos control y experimental (...). Mientras que toda hipótesis que difiere de una dada se llamará una hipótesis alternativa. Por ejemplo: Si una hipótesis es $\mathrm{p}=0,5$, hipótesis alternativa podrían ser $\mathrm{p}=0,7, \mathrm{p}$ " 0,5 ó $\mathrm{p}>0,5$.Una hipótesis alternativa a la hipótesis nula se denotará por $\mathrm{H} 1$.Al responder a un problema, es muy conveniente proponer otras hipótesis en que aparezcan variables independientes distintas de las primeras que formulamos. 


\section{Discusión}

No hay duda de que el uso de material didáctico facilita la enseñanza y constituye un elemento auxiliar en el proceso de aprendizaje funcionando como mediador en la educación de los estudiantes. Es por eso que deben utilizarse materiales didácticos que se presenten mediante la tecnología que es de uso común para los estudiantes, materiales innovadores bien pensados y fabricados especialmente para enseñar y aprender de manera que capten la atención de los estudiantes y les permitan llegar a adquirir determinados conceptos y contribuir así al desarrollo del pensamiento.

En el pre-test los y las estudiantes de ambos grupos tienen dificultades para entender la abreviación, la puntuación, los conectores y la coherencia con un 23,33\%, mientras que en el post-test se observa un gran incremento en la precisión en la escritura en los estudiantes del grupo experimental con 66,67 \% y en un mínimo incremento en el grupo control de $26,6 \%$ de acuerdo a los resultados presentados en cada uno de los ítemes. Es decir que la aplicación de la plataforma Edmodo es necesaria con el objetivo de que desarrollen la escritura académica.

Montolío (2002) la habilidad de escribir, frente a la del habla oral, constituye un aprendizaje, no es resultado de una capacidad innata: cualquier hablante es capaz de hablar su lengua de manera comprensible y coherente aunque no haya pisado jamás una escuela; sin embargo ningún hablante es capaz de escribir si no ha pasado por un periodo de formación en escritura (pág. 13).

Se observa que en el pre-test los y las estudiantes del grupo experimental tienen un bajo porcentaje al hacer un párrafo coherente mientras que el grupo control tiene un mínimo conocimiento en la elaboración de un párrafo coherente. Mientras que el grupo control tuvo un alto porcentaje en el desarrollo de ideas de un párrafo. Los estudiantes de los dos grupos tienen un bajo puntaje en la estructura de un párrafo, para lo cual se puede promover la comunicación y la transmisión de información virtual en los y las estudiantes de tercer semestre de la Carrera Inglés. En el post test los dos grupos tienen un incremento significativo en la escritura de un párrafo, en la estructura y en el desarrollo del mismo, pero en especial el grupo experimental ha mejorado en el proceso de la escritura cuando escribe párrafos.

Finalmente, los estilos de escritura los y las estudiantes de los dos grupos tienen un bajo rendimiento al escribir cartas formales e informales, un correo y un ensayo de acuerdo a los resultados del pre-test. Mientras que en el post test los y las estudiantes de los dos grupos incrementaron su rendimiento al escribir los diferentes estilos de escritura pero sin embargo el grupo experimental supero al grupo control, lo que significa que la integración de la teoría con la práctica en el desarrollo de la expresión escrita trajo muy buenos resultados con el uso de la plataforma Edmodo.

Con el uso de los distintos materiales didácticos a través de las TICs se puede brindar una educación de calidad a un mayor número de estudiante, la institución educativa debe estar preparada para ofrecer esta educación, con un equipo físico, técnico y tecnológico, capaz de afrontar los retos de la nueva era.

\section{Bibliografía}

Alonso, S., \& Palomares, A. (2013), Percepciones de la función tutorial en el Espacio. Europeo de Educación Superior del alumnado de la Facultad de Educación de Albacete. Universidad de Castilla-La Mancha, España. 
Arias, F., (1999) El Proyecto de Investigación, 3ra edición, Editorial Episteme, Caracas.

Cubo de Severino, L.,(2007) Discurso académico científico y perfil inferencial: el manual especializado. Tomado de http://aledar.fl.unc.edu.ar/files/Cubo-de-Severino-Liliana1.pdf

EF-EPI (2017) English Proficiency Index, $7^{\text {th }}$ edition, publishing by Learning Labs. Recuperado de https://www.ef.com.ec/

EF EPI (2017), El Inglés y la Tecnología. Obtenido de https://www.ef.com.ec/epi/insights/englishand-technology/

Gallardo \& Buleje, (2010) Importancia de las TICs en la educación Básica. Obtenido de http://revistasinvestigacion.unmsm.edu.pe/index.php/educa/article/view/4776/3850

García, P. L., \& Lacleta, M. L.-E. (2007). MOODLE: difusión y funcionalidades.

García S. et al (2015), Redes sociales Aplicadas a la Educación EDMODO.

Green (2000), New (1999) e Hirvela (2005), Escritura académica en entornos computacionales.

Hernández, J. (2010). Metodología de la Investigación, Quinta Edición, McGraw-Hill/ I interamericana Editores. México.

IBM SPSS Statistics (s/f), Obtenido de, https://www.ibm.com/es-es/marketplace/spss-statistics

Mallen, M. (2009). Proyecto de Investigación-Acción sobre un problema de Enseñanza del inglés, El uso de Herramientas Digitales en los Cursos Universitarios de inglés. México

Mar, M. (2000) Manual práctico de escritura académica, Volumen 3, Grupo Planeta.

Martínez, N., (s/f) Porque los estudiantes de las escuelas públicas no aprenden inglés. Obtenido de http://www.academia.edu/9741357/Por_qu\%C3\%A9_los_estudiantes_de_las_escuelas_p\%C 3\%BAblicas_no_aprenden_ingl\%C3\%A9s

Mejía, P. (2016), Plataforma Edmodo en el desarrollo de la escritura académica en los estudiantes de tercer semestre de la carrera inglés de la universidad central en el periodo 2015.

Montolío, E., et al (2002) Manual práctico de escritura académica, Volumen II.

Mumtag, (2005) El uso de material didáctico y las tecnologías de información y comunicación (TICs) para mejorar el alcance académico. Obtenido de http://www.palermo.edu/ingenieria/pdf2014/14/CyT_14_11.pdf

Monzón, V. (2013). Problemas en la enseñanza de lenguas. Universidad Autónoma de la Ciudad de México.

Paidós Ibérica, S.A. Gallardo, L. M., \& Buleje, J. C. (2010). Importancia de las TIC's en la Educación Básica Regular. Investigación Educativa vol. 14, 209-224.

Paz, M. (2013) Plataforma Edmodo versus Plataforma Moodle. Tomado de https://uvadoc.uva.es/bitstream/10324/3415/1/TFM-G\%20140.pdf

Pérez, C. (2013), El ensayo como herramienta en la enseñanza y el aprendizaje de la escritura académica, Argentina.

Piña, Emively, García, Faylenys, Govea, Lidia, estrategias de enseñanza basadas en un wiki para el desarrollo de la destreza escrita en estudiantes de inglés como lengua extranjera. Télématique [en linea] 2009, 8 (Sin mes) : [Fecha de consulta: 7 de diciembre de 2017] Disponible en:<http://www.redalyc.org/articulo.oa?id=78411787003> ISSN 1856-4194

Ramos, C. (2012) Prueba de Hipótesis, Obtenido de https://es.slideshare.net/crg110886/prueba-dehiptesis-12589447

Sánchez, M., Martínez, A., \& Hiracheta R., (2014) El uso de material didáctico y las tecnologías de información y comunicación (TIC's) para mejorar el alcance académico. Obtenido de http://www.palermo.edu/ingenieria/pdf2014/14/CyT_14_11.pdf

Sánchez R. (2015) t-Student. Usos y abusos. Obtenido de http://www.scielo.org.mx/scielo.php?script=sci_arttext\&pid=S0188-21982015000100009

Zorrilla (1993) Principales tipos de investigación. Obtenido de http://www.monografias.com/trabajos58/principales-tipos-investigacion/principales-tiposinvestigacion $2 . \operatorname{shtml}$ 